\title{
Geotritium: Implications of DeP Weak Interaction Fusion in Magmatic Systems
}

\author{
Thomas E. Ward \\ Techsource Incorporated, 20251 Century Blvd., Germantown, Maryland 20874, U.S.A
}

\begin{abstract}
Tritium in the environment comes from several sources: anthropogenic nuclear weapons and commercial nuclear reactors, atmospheric cosmic-ray production, and geotritium. Geotritium and its decay product ${ }^{3} \mathrm{He}$ is found in the earth's magmatic system evidenced by tritium emission from volcanoes and ${ }^{3} \mathrm{He}$ anomalies associated with MORB (Mid-Ocean Ridge Basalts), OIB (Oceanic Island Basalts) and deep mantle plumes. The ${ }^{3} \mathrm{He}$ can be accounted for assuming a radiogenic ${ }^{3} \mathrm{~T}\left({ }^{3} \mathrm{He}\right.$ ) component as opposed to a primordial source. It is proposed that the $d\left(p e^{-}, v\right)^{3} t$ weak interaction fusion (WIF) reaction $(\mathrm{Q}=5.474 \mathrm{MeV})$ is the source for both the geotritium and anomalous ${ }^{3} \mathrm{He}$ found in nature. The radiogenic lifetime for the WIF reaction, $d p+e^{-} \rightarrow{ }^{3} t+v_{e}$, in a metal hydride geologic environment, such as FeH/FeD in the core and mantle of the earth, was calculated to be $\tau_{\text {dep }}=1.61 \times 10^{9} y r$. The anomalous ${ }^{3} \mathrm{He}$ produced by the WIF reaction, a radio chronometer, was used to correlate and date mantle basalt processes deep within the earth. The $p\left(p e^{-}, v\right) d$ WIF reaction $(\mathrm{Q}=1.442 \mathrm{MeV})$ with a lifetime $\tau_{p e p}=2.54 \times 10^{12} y r$. was also examined as a possible geochronometer for the formation interval of the oceans, the duration of the earth's melting and degassing prior to the formation of the oceans. The formation age of the oceans was determined to be 3.93 billion years ago in good agreement with geologic magmatic activity and continental crust formation.
\end{abstract}

Key words: Geotritium, mantle helium, fusion, geochronology.

\section{Introduction}

Tritium is commonly found in the environment in the atmosphere, hydrosphere and within the earth itself, however its half-life (12.33 yr.) would seem to preclude timely geologic transport from the first two reservoirs into the mantle and ultimately back to the surface. The sources of tritium in the hydrosphere and atmosphere are mainly from anthropogenic nuclear weapons tests conducted prior to 1962, commercial nuclear reactors and cosmic-ray production in the atmosphere. In the earth, anomalous geotritium has been observed in volcanic lakes and volcanoes and correlated with ${ }^{3} \mathrm{He}$ anomalies associated with MORB (Mid Ocean Ridge Basalts), OIB (Oceanic Island Basalts) and deep mantle plumes [1-3]. As in the previous study the ${ }^{3} \mathrm{He}$ is accounted for assuming a radiogenic ${ }^{3} \mathrm{~T}\left({ }^{3} \mathrm{He}\right)$ component

Corresponding author: Thomas E. Ward, Ph.D., research field: nuclear science. produced in a WIF (weak interaction fusion) process, $d p+e^{-} \rightarrow{ }^{3} t+v_{e}, \quad$ a radioactive decay fusion process conducted in metal hydride mantle fluids and magma [1]. A radiogenic ${ }^{3} \mathrm{~T}\left({ }^{3} \mathrm{He}\right)$ component produced in the mantle of the earth can only be accounted for by assuming some form of nuclear reaction, i.e. radioactive decay [1], nuclear fission [4] or nuclear fusion [1-3, 5-6]. Although georeactors [4] are an appealing candidate given the history of the Oklo natural reactor, other fission evidence such as energy release and long-lived radioactive fission products that would accompany any radiogenic ${ }^{3} \mathrm{~T}\left({ }^{3} \mathrm{He}\right)$ to the surface is lacking. Additionally, conventional free space nuclear fusion reactions of interest here such as $p+d \rightarrow{ }^{3} \mathrm{He}+\gamma$, $d+d \rightarrow{ }^{3} t+n \quad$ and $\quad d+d \rightarrow{ }^{3} \mathrm{He}+p \quad$ are excluded since there is no evidence or acceptable theory these reactions occur in solids under conditions existing in the earth's mantle. However, electron capture radioactive decay such as $p p+e^{-} \rightarrow d+v_{e}$ 
and $d p+e^{-} \rightarrow{ }^{3} t+v_{e}$, termed WIF processes [1], can occur with very long lifetimes $\left(10^{9}-10^{12}\right) y r$ because: (a) the core and mantle temperature, pressure and particle densities are sufficiently high to allow a substantial geological reaction rate between the three particles in a metal hydride lattice matrix, and (b) the effect of the quantum tunneling through the coulomb barrier of the two charged protons in the presence of the electronic field (electrons) of a metal ion such as Fe largely cancels the Gamow penetrability factor allowing a low energy weak interaction nuclear reaction to occur. Notably, in the solar environment the WIF reactions, $p p+e^{-} \rightarrow d+v_{e}$ and $p p \rightarrow d+e^{+}+v_{e}$ are why the sun shines by providing deuteron fuel for nuclear fusion reactions in the sun.

The $d p+e^{-} \rightarrow{ }^{3} t+v_{e} \quad$ WIF reaction and subsequent tritium beta decay to ${ }^{3} \mathrm{He}$ is important with regard to a possible radio chronometric ${ }^{3} \mathrm{He}$ component that would allow dating basalt processes in geologic time. The question of whether radiogenic ${ }^{3} \mathrm{He}$ has contributed appreciably to the ${ }^{3} \mathrm{He} /{ }^{4} \mathrm{He}$ ratio anomalies observed between the atmospheric, oceanic seawater, MORB, OIB and deep mantel "hot-spot" plumes is an interesting and timely one (see Parman [7], commentary by Ladbury [8] and Anderson [9]). The standard accepted interpretation of helium isotopic anomalies reported in the literature over the past four decades has been based on a "primordial or protosolar" ${ }^{3} \mathrm{He}$ content of the earth's core and mantle [10-28]. The present interpretation of a radiogenic source of ${ }^{3} \mathrm{He}$ produced from geotritium decay to be used as a geochronometer of magmatic processes is both novel and controversial yet this new paradigm can be shown to resolve longstanding major differences between MORB, OIB and plume helium anomalies derived from the premise of the primordial ${ }^{3} \mathrm{He}$ concept [1, 7]. Additionally the new paradigm of a radiogenic ${ }^{3} \mathrm{He}$ component could lead to better defining the geochronology of the $\mathrm{D} / \mathrm{H}, \mathrm{U}$ and $\mathrm{Th}$ abundances in the continental crust [29] and in the differentiated upper and lower mantle (See references 1-13 in Ref. [18]).

\section{Mineral Physics, Lattice Vibrations and WIF Nuclear Reactions}

The solar lifetime for the $p p \rightarrow d+e^{+}+v_{e}$ and $p p+e^{-} \rightarrow d+v_{e}$ WIF reactions depend mainly on the density of protons and electrons, the Gamow penetrability factor and temperature in the sun [30, 31]. It is not at all obvious that $\mathrm{PeP}$ or $\mathrm{PeD}$ processes should proceed in low temperature geologic settings. However, theoretical optical model investigations into ultra-low energy two- and three-body nuclear interactions by Kim and Zubarev [32-34] indicate that a combination of Thomas-Fermi electronic shielding $[35,36]$ coupled with a very weak long range attractive imaginary (elastic + fusion) scattering potential allows for the Gamow Factor Cancellation, the GFC effect. Additionally, mineral physics of lattice vibrations thermally excited above the Debye temperature provide for particle-hole excitations within the electronic conduction band [37, 38] uniquely producing highly mobile protons and deuterons which share the electronic environment of the metal lattice with the electrons. It is important to note that the $p p \rightarrow d+e^{+}+v_{e}$ reaction does not require the electrons of $\mathrm{Fe}$ and therefore does not have a LRAI (long range attractive interaction) in the metal lattice. Without the assistance of the electronic Fermi contact potential in $\mathrm{Fe}$, the screening potential for the pp and pd reactions are only $43.4 \mathrm{eV}$. This "effective energy or temperature" is insufficient to produce the necessary GFC effect.

\subsection{GFC (Gamow Cancellation Factor)}

The Kim-Zubarev GFC mechanism [32-34] with a LRAI and in combination with a Fermi contact potential is used in a low energy nuclear reaction theory to investigate the $\mathrm{PeP}$ and $\mathrm{PeD}$ weak interaction decay lifetimes in the earth's early geological environment when melting, degassing and 
differentiation were occurring to create the first continental crust, oceans and atmosphere. The main premise is the earth's primordial hydrogen isotopes, chemically bound as iron hydrides in either the $\mathrm{Fe}$ core or the Fe-silicate mantle, decayed into deuterium or tritium (subsequently ${ }^{3} \mathrm{He}$ ) via WIF nuclear reactions during the time interval between the accretion of the earth 4.55 billion years ago and the degassing and formation of the early continental crust, MORB, oceans and atmosphere. The basic assumption is that proton and deuterium WIF reactions occur in the earth's Fe core or Fe-silicate mantel at thermal energies above the Debye temperature $(750 \mathrm{~K})$ and the rate of production of deuterium and tritium is largely determined by (1) the Thomas-Fermi electron screening energy of the protons and deuterons in $\mathrm{Fe}$, (2) the GFC effect and (3) the hydrogen isotope density. Details of the optical model formulation of ultra-low energy WIF fusion reactions can be found in Ref. [1] whereas a brief summary is provided by the following.

\subsection{Debye Temperature}

At temperatures above the Debye temperature $\left(\theta_{D}\right)$ of a metal or ceramic hydride, protons and deuterons becomes mobile as hole-state's with the electrons occupying the conduction band. The ceramic perovskites with oxygen vacancies created at elevated temperatures readily absorb water, followed by the hydroxyl groups filling the vacancies thus creating mobile protons and deuterons at temperatures above $\theta_{D}=750 K$ [39-44]. The high density and short 0-0 distance in MgSi Oxides favors hydration and proton migration. Thermally excited proton-electron particle-hole continuum states with hopping frequencies $>1 \mathrm{E}(+13)$ per sec are effectively developed by electronic screening potentials of the order of a $\mathrm{keV}$ in the vibrational lattice of Fe materials commonly found in geological settings, such as the earth's Fe core or in the mantle perovskites, $\mathrm{Mg}(\mathrm{Fe}) \mathrm{Si}$ Oxides.

\subsection{Screening Potential}

A key element in the theory is a LRAI in the imaginary scattering potential [32-34] resulting from the formation of an internal electric field $\left(E_{\text {int }}\right)$ in the metal ion lattice given by

$$
E_{\text {internal }}=-\nabla U_{s c}-\frac{\partial A_{c}}{\partial t}
$$

where $U_{s c}$ is the electronic screening potential and $A_{c}$ the Fermi Contact Potential for the electron-proton interaction at $\mathrm{r}=0[45,46]$, the LRAI. This form of an induced internal electric field is commonly known as a displacement potential.

The screening potential $U_{S C}$ is the Thomas Fermi screening energy [35, 36] given by

$$
U_{s c}=30.7 Z_{a} Z_{b}\left(Z_{a}^{2 / 3}+Z_{b}^{2 / 3}\right)^{1 / 2} e V
$$

with $Z_{a}=1$ and $Z_{b}=26$, yields a screening potential energy of $2.496 \mathrm{keV}$ for the three body system, $P e P$ or $P e D$, in the Fe or Fe-Silicate lattice. The screening potential is an "effective energy or temperature" for the PeP or PeD reactions to be used in the GFC formulation. The calculated value of 2.496 $\mathrm{keV}$ is greater than the equivalent central core temperature of the sun $(<1.35 \mathrm{keV})$.

\subsection{The Reaction Rates}

The PeP and PeD reaction rates in the earth core and mantle at "effective energies or temperatures" of $2.496 \mathrm{keV}\left(29 \times 10^{6} \mathrm{~K}\right)$ are comparable to the solar core, $1.293 \mathrm{keV}\left(15 \times 10^{6} \mathrm{~K}\right)$ and is given by:

$$
R_{p e p} \cong 1.102 \times 10^{-4}\left(\frac{\rho}{\mu_{e}}\right) T_{6}^{-1 / 2}\left(1+0.02 T_{6}\right) R_{p p}
$$

or reduced to,

$$
R_{p e p}=\chi_{p e p} R_{p p}
$$

where $\mu_{e}$ is the mean molecular weight per electron, $\rho$ the local proton or deuteron density and $\chi_{\text {pep }}$ is understood to represent the quantities in front of $R_{p p}$ in Eq. (3).

The proton-proton or proton-deuteron rate $\left(R_{p p}\right)$ is 
given by:

$$
R_{p p, p d}=\frac{n_{a} n_{b}}{1+\delta_{a b}}\langle\sigma v\rangle
$$

where $n_{a, b}$ are the particle densities, $1+\delta_{a b}$ is the Kronecker delta which prevents double counting and the average cross section times velocity is approximated by $\sigma \mathrm{V}$.

The comparison of the rates in the earth and sun reduces to the following equation:

$$
\begin{aligned}
& R_{\text {pep }}^{\text {earth }}=\frac{1}{1.01 \times 10^{10} y r}\left(\frac{\chi_{\text {earth }}}{\chi_{\text {sun }}}\right) x \\
& \left(\frac{n_{a b}^{e}}{n_{a b}^{s}}\right)\left(\frac{E_{\text {sun }}}{U_{s c}} e^{2 \pi \eta \frac{U_{s c}}{E_{s u n}}}\right)\left(\frac{v_{e}}{v_{s}}\right)
\end{aligned}
$$

The energies are converted to $T_{6}$ temperatures $U_{s c}=k_{B} T_{6}$, the mean molecular weight per electron (earth) $=2$, average mantle density $\rho_{\text {mantle }}=3.84 \mathrm{gcm}^{-3}$ and mantle proton particle density $n_{a b}=(1.44-1.65) \times 10^{22}$, equivalent to one conduction electron or particle-hole state per perovskite molecule, a $0.6 \%$ proton fraction, whereas for deuterons in the $\mathrm{PeD}$ reaction one uses $D / H=1.5 \times 10^{-4}$. The solar parameters were taken from Bahcall [30] (see his tables 4.4 and 4.5). The uncertainty is approximately $\pm 15 \%$ based on a $10 \%$ uncertainty in the overall PeP solar rate [30] and the range of mantle densities [37].

The calculated lifetimes are: (1) PeP WIF lifetime in the mantle of $\tau_{p e p}=(2.45 \pm 0.38) \times 10^{12} y r$ which is comparable to the lifetime in the sun of $\tau_{p e p}=4.34 \times 10^{12} y r$, and (2) PeD WIF lifetime in the mantle of $\tau_{p e d}=(1.61 \pm 0.18) x 10^{9} y r$. The PeD reaction lifetime is much shorter than the PeP lifetime due to the greater beta decay energy $(5.474 \mathrm{MeV}$ versus $1.442 \mathrm{MeV}$ ). The nuclear beta decay matrix elements are constant, the lifetime follows the well-known power law $\tau^{-1} \propto E_{\beta}^{-5.0}$. The PeD reaction in the earth is expected to be in secular equilibrium with the PeP reaction.

\section{Isotope Anomalies in the Mantle and Ocean}

During the evolution and development of a differentiated core, mantle and crust the molten earth degassed magmatic fluids that escaped to the surface of the earth to form the oceans and primitive atmosphere. The combined processes of core-mantle differentiation, degassing, ocean formation and heat loss cooled the earth allowing the mantle to evolve into cratons and primitive continental plates. The earth has a distinctive mode of planetary differentiation. Radioisotope dating of zircons using $\mathrm{Sr}, \mathrm{Nd}, \mathrm{Hf}$ and Pbhave established that early Archean mantle gneisses formed at about 3.9 Gyr followed by episodic mantle activity up to the present [7, 47-49]. The same is true for the evolution of the continental crust which covers about a third of the earth's surface today [49]. Studies of $\mathrm{D} / \mathrm{H}$ and ${ }^{3} \mathrm{He} /{ }^{4} \mathrm{He}$ isotopic ratios therefore provide a new chronometric tool set to study magmatic processes deep within the earth over geologic time.

\subsection{The PeP $\rightarrow D+v_{e}$ WIF Reaction and Formation} Age of the Oceans

The formation interval ( $\Xi$ ) between the accretion of the earth $4.556 \mathrm{Gyr}$ ago and the degassing and formation of the early oceans and atmosphere was calculated assuming the deuterium difference between seawater, $D / H=(1.5 \pm 0.1) \times 10^{-4}$, and the best estimate for proto solar gas, $D / H=(2.5 \pm 0.5) \times 10^{-5}$, or the measured Jovian atmospheric isotope ratio [50], $D / H=(2.6 \pm 0.7) \times 10^{-5}$, resulted from the PeP WIF reaction in the earth's early history. The formation interval equation is given as:

$$
\delta D / 2 H=\left(1-e^{-\lambda_{P e P} \Xi}\right)
$$

where $\lambda_{P e P}=\tau_{P e P}^{-1}, \quad \tau_{P e P}=(2.54 \pm 0.38) x 10^{12} y r$ and $\delta D / 2 H=(2.48 \pm 0.32) \times 10^{-4}$ using the Jovian measurement value [51] yields, $\Xi=(630 \pm 160) x 10^{6} y r$, where half of the 
estimated uncertainty is from the measured uncertainties in the $\mathrm{D} / \mathrm{H}$ ratios and the remainder from the pep lifetime calculation [30]. The formation age of oceans can be estimated using the accretion age of the solar system of $(4.556 \pm 0.002) \times 10^{9} y r$ [52] and the formation interval of $\Xi=(630 \pm 160) \times 10^{6} y r$ yielding $(3.93 \pm 0.16) \times 10^{9} y r$ before the present. This formation age agrees well with the appearance of the first ancient continental crust or cratons and other geologic evidence such as the water-lain sediments dated approximately to 3.8 Gyr ago [53].

3.2 The DeP $\rightarrow{ }^{3} T+v_{e}$ WIF Reaction and Helium Anomalies

Tritium concentrations in excess of atmospheric and seawater values have been observed in magmatic fluids and emanations from volcanic lakes and hot spot volcanoes such the Kilauea (Hawaii) and Alcedo (Galapagos) [1, 2, 5]. The explosive massive eruption of Mt Ulu (Kilauea) on Hawaii in 1972 measured at an air monitoring station 100 miles away on Maui was found to have released more than 5000-Ci of tritium or a concentration of about 90 tritium atoms per liter of air [54]. The anomalous amount of tritium could not be accounted for from sources of tritium in seawater or meteoritic ground water. Likewise, tritium in the lowest layers of volcanic lakes in Europe and Turkey have been shown to store excess anomalous tritium concentrations derived from mantle fluids and volatiles [2]. The presence of excess tritium in these varied magmatic systems provides strong evidence of WIF reactions taking place in the mantle [1].

Comparing ${ }^{3} \mathrm{He} /{ }^{4} \mathrm{He}$ ratios for average MORB $\left(R / R_{a}=8 \pm 1\right)$ with the range of values of OIB $\left(R / R_{a}\right.$ $=15-40$ ) one could infer that the upper mantle has been degassed and MORB represents a depleted upper mantle with a depth of less than $670 \mathrm{~km}$. Here $\left(R_{a}=1.39 \times 10^{6}\right)$ is the atmospheric He ratio. On the other hand, the increased He ratio for OIB and deep mantle plumes seem to indicate source's from the lower mantle with a much longer residence time.
The lower mantle may be isolated from the upper portions over periods of several billion years [51, 55-57]. If that is the case, then WIF reactions considered here could provide a new radiogenic point of view. Assuming the difference in helium ratios of the plumes and MORB is not due to $\mathrm{U}, \mathrm{Th}$ and $\mathrm{D} / \mathrm{H}$ concentration changes but rather that MORB is degassed whereas the deep mantle is not, then one can calculate the increased concentration of radiogenic ${ }^{3} \mathrm{He}$ over the past $4.55 \mathrm{Gyr}$ in the deep mantle. The maximum OIB/plume to MORB ratio is calculated to be $R_{O I B} / R_{M O R B}=(6.5 \pm 1.6)$ using the WIF lifetimes. Correcting for the decay of $U$ and Th over the last $4.55 \mathrm{Gyr}$ yields an OIB/plume ratio $R_{O I B}=(53 \pm 13)$, a value in good agreement with what is observed experimentally.

In Fig. 1 is displayed a large compilation of experimental He ratios for MORB and OIB obtained from the literature by Parman [7].

The green and orange fields are zircon age distributions of the corresponding MORB and OIB helium ratios $\left(R^{-1}\right)$. In the model presented above the isolated deep mantle contributes to OIB and plumes

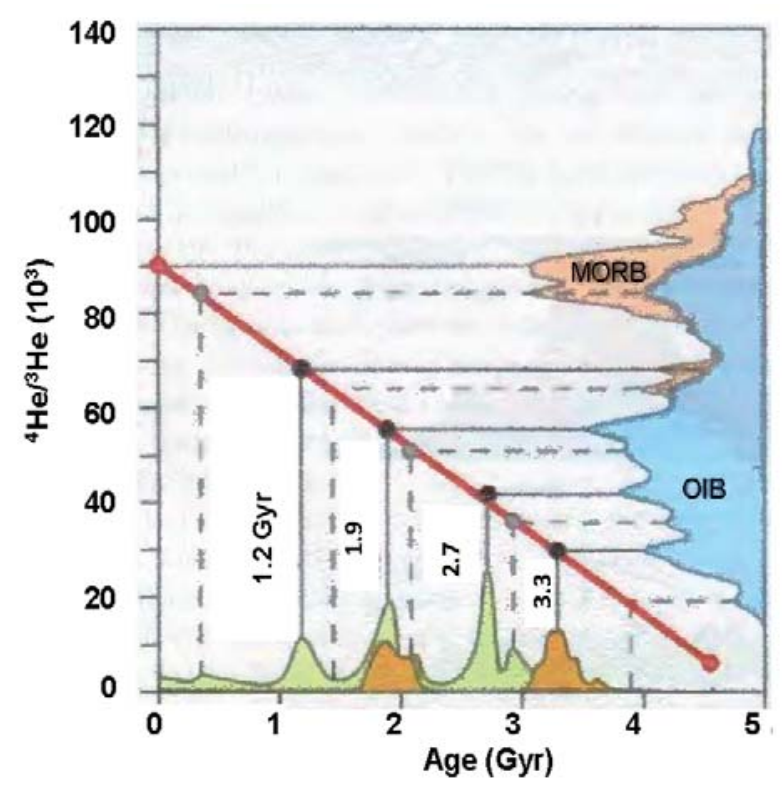

Fig. 1 Chronological development of MORB and OIB. The green and orange fields are zircon age distributions of the corresponding MORB (red) and OIB (green). This figure was adapted from Parman [7]. 
with the upper mantle contributing to MORB. In the chronological plot the experimental red line has a lifetime of $\tau=(1.63 \pm 0.07) G y r$ which compares well with the calculated PeD WIF lifetime $\tau_{P e D}=(1.61 \pm 0.18) G y r$. Note that the estimated amount of geotritium residing in secular equilibrium in the mantle is about 1 trillion $\mathrm{Ci}$ or about 100 metric ton.

Some important conclusions of the Parman study [7] include: (a) the helium isotopes in OIB appear to preserve the mantles depletion history, (b) the helium evolution does not require a primitive or primordial mantle reservoir, (c) a correspondence between ages of mantle depletion events and crustal formation are provided by helium ratios in OIB, and (d) the helium ratios are directly proportional to the time at which sources were formed so they can be directly compared to crustal age growth. These conclusions support the present study assigning tritium and subsequent helium production in the earth's mantle to radiogenic processes accounted for in PeD WIF reactions.

\section{Conclusion}

Evidence that the WIF process is responsible for helium anomalies in magmatic systems is provided by the good agreement of the calculated lifetime for the PeD WIF reaction with the lifetime of the chronological plot in Fig. 1. This new paradigm of a radiogenic ${ }^{3} \mathrm{He}$ component provides a new radioactive geochronometer to date mantle processes over the lifetime of the earth.

\section{Acknowledgement}

The author would like to thank Professor Y. E. Kim for valuable conversations and collaborations over many years. This work was made possible by financial support from Techsource Incorporated and the US Department of Energy Office of Nuclear Energy Office under contract number NE-4091.

\section{References}

[1] Ward, T. E. 2000. “The Possible Role of PeP Weak
Interactions in the Early History of the Earth.” In Origin of Elements in the Solar Systems: Implications of Post-1957 Observations, edited by Manual, O. Kluwer Academic/Plenum Publishers.

[2] Jiang, S., and He, M. 2010. Naturwissenshaften 97: 655.

[3] Jiang, S., and He, M. 2012. Plasma Science and Technology 14: 438.

[4] Herndon, J. 2003. PNAS 100: 3047.

[5] Palmer, E. 1991. "Nuclear Fusion in the Earth." "Anomalous Nuclear Effects in Deuterium/Solid Systems, Provo, Utah 1990.” AIP Conference Proceedings 228: 616.

[6] Sheely, E. 1991. "Examination of the Possibility of Cold Nuclear Fusion Occurring with the Earth's Mantle.” "Anomalous Nuclear Effects in Deuterium/Solid Systems, Provo, Utah 1990.” AIP Conference Proceedings 228: 646.

[7] Parman, S. 2007. Nature 446: 900.

[8] Ladbury, R. 1999. "Model suggests deep-mantel topography goes with the flow.” Physics Today 52 (8): 21-4.

[9] Anderson, D. 2000. International Geological Review 42: 289.

[10] Clarke, W., Beg, M., and Craig, H. 1969. Earth Planet Sci. Lett. 6: 213.

[11] Craig, H., Clarke, W., and Beg, M. 1975. Earth and Planet Sci. Lett. 26: 125.

[12] Lupton, J., and Craig, H. 1975. Earth Planet Sci. Lett. 26: 133.

[13] Kurz, M., Jenkins, W., and Hart, S. 1982. Nature 297: 43.

[14] McKenzie, D., and O’Nions, R. 1983. Nature 301: 229.

[15] Kaneoka, I. 1983. Nature 302: 698.

[16] Allegre, J., et. al. 1983. Nature 303: 762.

[17] O’Nions, R., andOxbugh, E. 1983. Nature 306: 429.

[18] Jochum, K., et al. 1983. Nature 306: 431.

[19] Porcelli, and Wasserburg, G. 1995. Geochim. Cosmochim. Acta 59: 4921.

[20] Rocholl, A., et al. 1996. Geochim. Cosmochim. Acta 60: 4773.

[21] Patterson, D., Farley, K., and Mclnnus, B. Geochim. Cosmochim. Acta 61: 2485.

[22] Niedermann, S., Bach, W., and Erzinger, J. 1997. Geochim. Cosmochim. Acta 61: 2677.

[23] Hoffmann, A. 1997. Nature 385: 219.

[24] Kamijo, K., Hashizume, K., and Matsuda, J. 1998. Geochim. Cosmochim. Acta 62: 2311.

[25] Eiler, J., Farley, K., and Stolpher, E. 1998. Geochim. Cosmochim. Acta 62: 1977.

[26] Zhang. 1998. Geochim. Cosmochim. Acta 62: 3185.

[27] Hanyu, T., Kaneoka, I., and Nagao, K. 1999. Geochim. Cosmochim. Acta 63: 1181.

[28] Pedroni, A., Hammerschmidt, K., and Freidricksen, H. 
1999. Geochim. Cosmochim. Acta 63: 515.

[29] Wedepohl, K. 1995. Geocvhim. Cosmochim. Acta 59: 1217.

[30] Bahcall, J. 1989. Neutrino Astrophysics (Chapter 3). New York, NY: Cambridge University Press, 567.

[31] Clayton, D. 1968. Principles of Stellar Evolution and Nucleosynthesis (chapter 4 and 5). New York, NY: McGraw Hill, 612.

[32] Kim, Y., and Zubarev, A. 1995. Few-Body Systems Suppl. 8: 334.

[33] Kim, Y., and Zubarev, A. 1996. Nuovo Cimento Al08: 1009.

[34] Kim, Y., and Zubarev, A. 1996. "Neutrino’ 96, Proc. 17th International Con/On Neutrino Physics and Astrophysics." Edited by Enqvist, K., Huitu, K., and Maalanpi, J. World Scientific: $120-6$.

[35] Lindhard, J., Nielsen, V., and Scharff, M. 1968. Mat. Phys. Medd. Dan. Vid Selsk 36: 47.

[36] Zeigler, J., Biersach, J., and Littmark, U. 1995. The Stopping and Range Ions in Solids. New York, NY: Pergamon Press, 128.

[37] Poirier, J.-P. 1991. Introduction to the Physics of the Earth's Interior. New York, NY: Cambridge University Press, 23-37.

[38] Kittel, C. 1986. Introduction to Solid State Physics. 6th edition. New York, NY: John Wiley and Sons, 108-43.

[39] Navrotsky, A. 1999. Science 284: 1788.

[40] Kreuer, K. 1996. Chem. Mater. 8: 610.
[41] Kreuer, K. 1997. Solid State Ionics 91: 1.

[42] Norby, T. 1990. Solid State Ionics 40: 857.

[43] Nowick, A., and Du, Y. 1995. Solid State Ionics 11: 137.

[44] Bose, K., and Navrotsky, A. 1998. J. Geophys. Res. 103: 9713.

[45] Yosida, K. Theory of Magnetism. 1996. Berlin/Heidelberg, Germany: Springer-Verlag, 13-5.

[46] Low, F. 1997. Classical Field Theory. New York, NY: John Wiley and Sons, 24-66.

[47] Bennett, V. 2005. "Compositional Evolution of Mantle." in The Mantle and Core, Chapter 13, edited by Carlson, W. Treatise on Geochemistry Vol. 2. Elsevier.

[48] Meyzen, C., et al. 2007. Nature 447: 1069.

[49] Hawkesworth, C., and Kemp, A. 2006. Nature 443: 811.

[50] Niemann, H., et al. 1996. Science 272: 846.

[51] Kerr, R. 1999. Science 283: 1826.

[52] Luair, G., and Shukolyukov, A. 1998. Geochim. Cosmochim. Acta 62: 2863.

[53] Titayeva, N. 1994. Nuclear Geochemistry. Moscow, Russia: MIR Publishers, CRC Press, 98-102.

[54] Ostlund, H., and Mason, A. 1984. Atmospheric Tritium 1968-1984. Tritium Laboratory Report No. 14, University of Miami, Florida.

[55] van der Hilst, R., and Karason, H. 1999. Science 283: 1885.

[56] Kellogg, L., Hager, B., and van der Hilst, R. 1999. Science 283: 1881.

[57] Kaneshima, S., and Helffrich, G. 1999. Science 283: 1888. 